116. Zaborowski: Experimentelle Untersuchungen über die Regeneration der quergestreiften Muskeln. Arch. f. exp. Path. u. Pharm., 1889, Bd. 25, S. 415 , und In.-Diss. Genf 1889.

117. Zabn: Die degenerativen Veränderungen der Zwerchfellmuskulatur, ihre Ursachen und Folgen. Dieses Arch., 1878, Bd. 73, S. 166.

118. Zenker: Über die Trichinenkrankheit des Menschen. Dieses Archiv, 1870 , Bd. 18, S. 561 .

119. Derselbe: Über die Veränderungen der willkürlichen Muskeln im Typhus abdominalis. Nebst einem Exkurs über die pathologische Neubildung quergestreiften Muskelgewebes. Leipzig 1864. Vorl. Mitt. Jahresber. der Ges. f. N. u. H. f. 1858-1860. Dresden 1861, S. 49, und Beilage zum Tageblatt der 36. Yers. D. Nat.

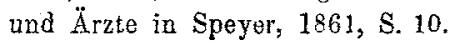

\title{
VIII. \\ Über die reine Mediaverkalkung der Extremitätenarterien und ihr Verhalten zur Arteriosklerose.
}

(Aus dem Pathologisch-anatomischen Institut des Allgem. Krankenhauses Hamburg-Eppendorf.)

Von

Dr. J. G. Mönckeberg.

Die Verkalkung peripheriseher Gefäße als isolierter Prozeß, d. h. ohne endarteriitische Veränderungen an Ort und Stelle, wird in den Lehrbüchern ein seltenes Vorkommnis genannt und den im vorgeschrittenen Alter auch an anderen Organen des Körpers auftretenden Kalkablagerungen zugerechnet. Nach Marchand ${ }^{1}$ sind bei diesem Prozeß die Arterien mit muskalärem Typus, namentlich die der untern Extremität bevorzugt. Überhaupt wird von den Autoren betont, daß die Verkalkung namentlich in der Tunica media vor sich geht, während isolierte Kalkherde in den anderen Wandschichten öfter als Teilerscheinung der Arteriosklerose zu beobachten sind. Marchand knüpft an seine Beschreibung der Mediaverkalkung die Vermutung an, daß es sich hierbei möglicherweise um eine Erkrankung sui 
generis handele. Jedenfalls ist pathologisch-anatomischerseits die Ansicht vorherrschend, daß die isolierten Kalkablagerungen in der Media peripherischer Gefäße nichts direkt mit arteriosklerotischen Veränderungen zu tun haben, wie denn auch $0 \mathrm{rth}^{2}$ ausdrücklich davor warnt, von der Verkalkung der Arterien ohne weiteres auf eine bestehende Endarteriitis deformans zu schließen.

Trotzdem wird dieser Prozeß von klinischer Seite immer and immer wieder entweder einfach mit der Arteriosklerose identifiziert oder man stellt aus der starren, fragilen Beschaffenheit fühlbarer Arterien die Diagnose auf eine Sklerose anderer Gefäße, die nicht der Palpation zugänglich sind. So schreibt z. B. Strümpell ${ }^{3}$ in seinem Lehrbuch: "Um am Lebenden zu entscheiden, ob eine Arteriosklerose vorhanden ist, sind wir selbstverständlich ausschließlich auf die Untersuchung einiger peripherischer, der Betastung zugänglicher Arterien angewiesen"; und an anderer Stelle: "Namentlich an der Radialis hat man bei starkem Atherom das Gefühl, als wenn man eine Gänsegurgel anfaßt". In einer ganzen Reihe von Fällen scheint der Schluß von der Verkalkung peripherischer Arterien auf eine Arteriosklerose innerer Gefäße zweifellos seine Berechtigung zu haben, wie man sich leicht bei den Obduktionen der unter der Diagnose Arteriosklerose eingelieferten Leichen überzeugen kann. Doch sieht man andererseits wieder Fälle, bei denen intra vitam aus der Beschaffenheit der Extremitätenarterien dieselbe Diagnose gestellt war, wo überhaupt arteriosklerotische Prozesse im innern Organismus nicht nachzuweisen sind oder so minimale Grade zeigen, daß sie unmöglich klinisch in die Erscheinung getreten sind. Dies gibt übrigens Strüm pell auch zu, indem er schreibt: „Wenn wir auch an den genannten Arterien das Atherom häufig direkt und sicher nachweisen können, so ist doch hieraus der Schluß auf ein gleichzeitiges Atherom der inneren Arterien stets nur mit Vorsicht zu ziehen."

Um nun zu prüfen, inwieweit ein Zusammenhang zwischen der Mediaverkalkung der Extremitätenarterien und der Arteriosklerose der Aorta, der Coronar- und der basalen Hirnarterien besteht, habe ich auf Veranlassung meines Chefs, Herrn Dr. E. Fränkel, im Laufe eines Jahres aus dem Material des hiesigen Institutes bei einer Anzahl von Fällen reiner Mediaverkalkung 
peripherischer Arterien das Gefäßsystem einer genauen Untersuchung unterzogen. Ich berïcksichtigte dabei einerseits hauptsächlich die Gefäßabschnitte, welche eine Palpation am Lebenden gestatten, andererseits diejenigen inneren Gefäße, deren Erkrankung klinisch am häufigsten von Wichtigkeit ist (Aorta, Coronar- und basalen Hirnarterien). Außerdem sammelte ich eine zweite Reihe von Fällen mit Arteriosklerose peripherischer Arterien, und schließlich Fälle ohne makroskopisch sichtbare Veränderungen an den der klinischen Untersuchung zugänglichen Arterien. Im ganzen beläuft sich mein Material auf 130 Fälle, von denen in 86 Fällen genauere mikroskopische Untersuchungen vorliegen. Dabei wurde $55 \mathrm{mal}$ die anatomische Diagnose auf reine Mediaverkalkung der Extremitätenarterien mit oder ohne Arteriosklerose innerer Gefäße gestellt; $18 \mathrm{mal}$ dehnte sich die Arteriosklerose auf die peripherischen Arterien aus, wobei in zehn Fällen auch Kalkablagerungen in der Media mit bloßem Auge zu erkennen waren. In den übrigen 57 Fällen konnte bei bestehender Arteriosklerose innerer Arterien an den Extremitätengefäßen nichts abnormes konstatiert werden. Ich lasse zunächst die $\mathrm{Be}$ funde bei der reinen Mediaverkalkung folgen.

I. Die reine Mediaverkalkung peripherischer Arterien.

Nach den Autoren gehen der Ablagerung von Kalksalzen in der Media degenerative Prozesse voraus, die namentlich in einer Verfettung, seltener in hyaliner Degeneration der Muskelelemente bestehen. Die Verfettung ist in vorgeschritteneren Fällen schon dem bloßen Auge sichtbar. Man kann sie oft in der Femoralis beobachten; nach Freilegung der Gefäße am Oberschenkel sieht man an ihr von außen schmale, gelbliche Streifen, deren Längsachse parallel zum Verlauf der zirkulären Muskelfasern gestellt ist; nach dem Aufschneiden der Femoralis scheinen dieselben Flecke deutlich durch die glatte Intima durch. Mikroskopisch kann man sich nach voraufgegangener Fixierung in Flemming scher Lösung leicht davon überzeugen, daß eine partielle Verfettung der zirkulären Muskelfasern in Gestalt feinster Tröpfchen stattgefunden hat. Die Kalksalze werden nach der herrschenden Ansicht zunächst in Form feiner Körnchen in und an die degenerierten Muskelfasern abgelagert. An frischen Schnitten er 


\section{4}

scheinen diese Körnchen ziemlich stark lichtbrecbend, nach Härtung und Färbung mit Hämatoxylin intensiv blau. Dieses Stadium habe ich rein nur ein einziges $\mathrm{Mal}$ an einer Iliaca communis beobachtet, und da war es makroskopisch auch nicht erkannt worden. Dagegen kann man es häufig sehen, gepaart mit vorgerückteren Stadien und außerdem in derMedia bei endarteriitischen Prozessen. Mit dem bloßen Auge wird sich dieser Grad der Kalkablagerung kaum von der einfachen Degeneration unterscheiden lassen, dagegen kann ein feines Knirschen beim Durchschneiden die Anwesenheit von Kalk verraten. Nachdem durch Vermehrung der Abscheidung die Kalkkörnchen immer dichter zusammengelagert. sind, verschmelzen sie allmählich zu Bälkchen und Spangen, die breiter oder schmäler das Lumen des Gefäßes in größerer oder geringerer Ausdehnung umfassen. Bei diesem "Zusammenfließen" (Marchand) der Kalkkörnchen findet scheinbar an den betreffenden Stellen eine Abnahme der Dicke der Media statt; diese scheinbare Reduktion kommt dadurch zustande, daf die Verkalkung an degenerierten, also nicht mehr kontraktionsfähigen und erschlafften Muskelfasern vor sich gebt. Das siebt man bereits bei makroskopischer Betrachtung: von außen hat das Gefäß nicht' mehr die normale glatte Oberfläche; die opak weißgelben, verkalkten Partien buchten sich vor, während die unberührt gebliebenen eingesunken erscheinen. Nach dem Aufschneiden bemerkt man, daß die den verkalkten Bälkchen und Spangen entsprechenden Stellen unter dem übrigen Niveau der Innenfläche liegen. Sehr deutlich treten diese Verbältnisse auch an Röntgenbildern solcher Gefäße in die Erscheinung.

Marchand hat schon darauf hingewiesen, daß diese Niveaudifferenzen durch eine Kontraktion der nicht verkalkten, also noch funktionstüchtigen Teile der Media zustande kommen. Man kann dieses Verhalten direkt dadurch beweisen, daß man unter Druck in das betreffende Gefäß irgend eine geeignete Injektionsmasse spritzt und dieses dann so konserviert. Bei nachträglichem Aufschneiden findet man die Niveaudifferenzen ausgeglichen bis auf einige kleine Erhabenheiten, die durch die zackige Begrenzung der Kalkstreifen hervorgerufen werden. Außerdem spricht aber auch das Verhalten der Elastica interna. für die Richtigkeit dieser Angabe: Nimmt man den oben ge- 
schilderten Injektionsversuch an einem normalen Gefäße vor und macht dann mikroskopische Schnitte, so findet man die Elastica interna völlig gestreckt verlaufend, wie Ernst ${ }^{4}$ und Matusewicz ${ }^{5}$ es für das Verhalten der elastischen Innenmembran intra vitam angegeben haben. Ebenso sieht man die Elastica interna gestreckt über die verkalkten Partien der Media verlaufen, während sie an den unverkalkten Stellen die bekannte halskrausenartige Fältelung zeigt. Dies spricht dafür, daß die verkalkten Partien gleichsam in Vasodilatation erstarrt sind, während die übrigen Teile der Media die in und nach dem Tode erfolgende Kontraktion aller Arterien des Körpers mitgemacht haben. Das Verhalten der Elastica interna spricht andrerseits gegen die Ernst-Matusewiczsche Behauptung. Denn die Gefäßweite ist im lebenden Organismus fortwährenden Schwankungen unterworfen, entsprechend den Phasen der Pulswelle und der Reizung der Gefäßnerven. Die Behauptung ist demnach so zu modifizieren, daß die elastische Innenmembran intra vitam bei Vasodilatation gestreckt, bei Vasokontraktion gefältelt verläuft. An normalen Arterien von Leichen findet sich stets überall die Fältelung infolge der oben erwähnten Kontraktion, die das Blut aus den Arterien in die Venen treibt.

Im lebenden Organismus werden also die Niveaudifferenzen. der Innenfläche bei der Mediaverkalkung sich auf dem Höbepunkt der Pulswelle und bei Reizung der Vasodilatatoren ausgleichen, während sie in den übrigen Phasen in geringerem oder gleichem Grade wie an der Leiche vorhanden sind. Diese vitalen Verhältnisse sind von Wichtigkeit bei den später zu besprechenden Intimaveränderungen, die sich im Anschluß an die Mediaverkalkung entwickeln.

Mikroskopisch kann man konstatieren, daß die Verschmelzung der abgelagerten Kalksalze zunächst in den mittleren Schichten der Media vor sich geht. Man sieht auf Querschnitten mit Hämatoxylin dunkelblau gefärbte Streifen in der Mitte der Media, die zunächst meist nur einen geringen Teil der Circumferenz einnehmen und an ihren Enden besenartig aufgesplittert erscheinen. Diese büschelförmigen Endpartien entsprechen dem vorher geschilderten Körnchenstadium der Kalkablagerung. Hier liegen feinere und gröbere Kalkpartikel in und an den Muskelelementen. 
An den verkalkten Stellen macht die Elastica interna einen Bogen nach außen und das Lumen zeigt hier eine Ausbuchtung. Die Elastica interna verläuft, wie gesagt, über die verkalkten Partien gestreckt; häufig sieht man an ihr eine Aufsplitterung in mehrere Lamellen, von denen die äußerste und innerste am stärksten sind. Ist diese. Teilung vorhanden, so findet man zaweilen das innere Blatt halskrausenartig, das äußere gestreckt verlaufend, während die zwischen beiden liegenden elastischen Elemente körnige Degenerationsstadien aufweisen; manchmal. ist schon bei diesem Verkalkungsstadium eine Fragmentation des äußeren Blattes der Elastica zu bemerken. Mehrmals sah ich bei Hämatoxylin-Eosin-Färbung feinste Kalkkörnchen an die elastische Membran angelagert, während ich nie eine richtige Verkalkung bei ihr konstatieren konnte. Die übrige Media zeigt weder an den muskulären, noch an den elastischen Bestandtheilen Bemerkenswertes. An Intima und Adventitia braucht in diesem Stadium noch nichts abnormes vorhanden zu sein; doch finden sich bisweilen bereits Verhältnisse, die ich weiter unten schildern werde. An vorgerückteren Stellen sieht man, daß allmählich auch die inneren und dann die äußeren Schichten der Media in die Verschmelzung eingezogen werden, bis schließlich zwischen Elastica interna und externa stellenweis nur Kalk zu sehen ist. Die Kerne behalten in diesen verkalkten Partien noch auffallend lange ihre Tinktionsfähigkeit; außerdem lassen sich meist die einzelnen verschmolzenen Muskelelemente noch nachweisen, so daß die Kalkablagerung mikroskopisch streifig aussieht. Außer dieser in und an den zirkulären Muskelfasern stattfindenden Kalkablagerung sieht man häufig auf Querschnitten kleine rundliche oder eckige Herde meist direkt an der Elastica interna oder zwischen den aufgesplitterten Blättern dieser Membran oder auch in der Nähe der Elastica externa. Die elastischen Elemente ziehen entweder dicht an ihnen vorbei oder sie treten auch durch die Herde hindurch, wobei sie ihre völlig normale Tinktionsfähigkeit und ihre glatte, scharfe Konturierung behalten; bisweilen sieht man eine Fragmentation der Elastica innerhalb eines solchen kleinen Herdes. Äbnlich verhalten sich auch oft die intermuskulären elastischen Fasern der Media bei der Verkalkung; auch sie sind manchmal färberisch noch klar und scharf 
in den verkalkten Partien za differenzieren, wenn selbst von den Kernen der Maskelzellen nichts mehr zu sehen ist. Andererseits finden sich in manchen Fällen zwischen den verkalkten mittleren Fasern der Media und der Elastica interna elastische Elemente, die deutlich die von Dmitrijeff ${ }^{6}$ angegebenen Formen der Degeneration zeigen. Daß die kleinen Kalkherde quergetroffene verkalkte Längsfasern darstellen, ist unwahrscheinlich, einmal wegen ihres Sitzes und dann, weil man an denselben Stellen auch auf Längsschnitten Herde von gleicher Form findet. Sie entsprechen wahrscheinlich frei im Gewebe liegenden Kalkab. lagerungen.

Die Verkalkung nimmt später immer größere Dimensionen an, die einzelnen Spangen und Streifen verbinden sich zu halben und ganzen Ringen, und die Arterie gewinnt das bekannte "gänsetracheaartige" Aussehen, indem die verkalkten Ringe von außen über die unversehrten $Z$ wischenpartien prominieren. Nach dem Aufschneiden solcher Arterien sieht man auf der Innenfläche außer den bereits erwähnten Niveauunterschieden und abgesehen von Vorbuchtungen, die durch die Enden beim Auseinanderbiegen zerbrochener Kalkringe entstanden sind, noch rundliche oder längliche Erhabenheiten von harter Konsistenz, die, wie das Mikroskop zeigt, Kalkherden in der Media entsprechen. Diese sind die stark vergrößerten runden oder eckigen Herdchen an den elastischen Lamellen des vorigen Stadiums, die nunmehr einen großen Teil oder den ganzen Durchmesser der Media einnehmen und die Elastica interna entweder stark gegen das Lumen vorbuchten oder sie bereits durchbrochen habon und in die Intima eingedrangen sind. Die Herde haben ein stark zerklüftetes Aussehen, ähnlich dem, wie man es in verkalkten atheromatösen Herden anzutreffen pflegt. Durch das reichliche Vorhandensein solchèr rundlicher Kalkablagerungen kommt es, daß man fast nie auf Quersohnitten einen richtigen, überall annähernd gleich breiten Mediakalkring zu sehen bekommt; fast immer wird der Ring an einer oder mehreren Stellen von solchen runden Herden unterbrochen, ja er kann geradezu wie ein Rosenkranz aussehen.

In diesem Stadium trifft man in den meisten Fällen auch mehr oder minder hochgradige Veränderungen in den andern 
Wandschichten an. Zunächst findet eine Wucherung der Intima statt und zwar an den Stellen, welche infolge starker Verkalkung der Media an der Leiche am meisten unter dem Lumenniveau liegen. Diese Niveaudifferenz wird, wie ich oben schon hervorgehoben, intra vitam nur auf der Höhe der Pulswelle und bei Vasodilatation ausgeglichen, während sie in den übrigen Pulsphasen auch vital vorhanden ist. Hier bestehen also Verhältnisse in der Gefäßwand, welche genau denjenigen entsprechen, die Tho ma ${ }^{7}$ für das Zustandekommen der Arteriosklerosis nodosa verantwortlich gemacht hat. Besteht also Thomas Theorie zu recht, so muß unbedingt die Folge der mit der Degeneration und Verkalkung Hand in Hand gehenden partiellen Dilatation der Gefäßwand eine reparatorische Intimawucherung sein. Und, wie gesagt, findet man auch tatsächlich oft eine die Niveaudifferenzen ausgleichende Verdickung der Intima, an der sich hauptsächlich das elastische Gewebe beteiligt, während das Bindegewebe weniger hervortritt. Doch erhält die Thomasche Theorie von der Notwendigkeit einer Intimawucherung bei partiell fehlender Elastizität der Media dadurch einen Stoß, daß Fälle von schwerer Mediaverkalkung vorkommen ohne diese sekundäre Wucherung der Intima. Und abgesehen von diesen Fällen, von denen in meinem Material sich eine ganze Reihe findet, ist die bei der Mediaverkalkung auftretende Intimawacherung im Gegensatz zur Arteriosklerose dadurch charakterisiert, daß sich nie degenerative Prozesse an die Neubildung von elastischen Fasern und Bindegewebe anschließen, daß es nie hier zum Atherom kommt. Die sekundären Veränderungen in der Intima hat Marchand bereits erwähnt; derselbe Autor hat auch in einer Abbildung das Verhalten der Elastica interna über verkalkten Partien der Media zum Ausdruck gebracht (a. a. 0. Fig. 9 B).

Die Veränderungen, die man in der Adventitia beobachtet, betreffen zumeist die Vasa vasorum, die mehr oder minder hochgradige Intimawucherung zeigen. Man hat als Erklärung der degenerativen und zur Kalkablagerung führenden Vorgänge in der Media eine mangelhafte Ernährung der Gefäßwand verantwortlich gemacht. Ich muß es dahingestellt sein lassen, ob die erwähnte Erkrankung der Vasa vasorum das Primäre ist: ich 
sah sie nur vereinzelt bei beginnender Mediaverkalkung, während die Mehrzahl der Fälle mit Intimawucherung der Vasa vasorum vorgeschritteneren Stadien angehören. Aber auch in diesen sind sie nicht konstant nachzuweisen. $\mathrm{Zu}$ berücksichtigen dabei ist, daß bei der Kleinheit der Vasa an peripherischen Gefäßen es überhaupt zuweilen unmöglich ist, Erkrankungen an ihnen zu konstatieren, zumal wenn sie kein Blut enthalten. Außer den Erkrankungen der Vasa sieht man häufig eine starke Wucherung des elastischen Gewebes der Adventitia nnd eine hyaline Degeneration des Bindegewebes. Die begleitenden Venen zeigen in einer großen Zahl meiner Fälle endophlebitische Veränderungen.

In den höchsten Graden der Mediaverkalkung ist die Arterie in ein starres Rohr verwandelt. Nimmt man eine größere Strecke von solchem Gefäß aus der Leiche heraus, so kann man an dem einen Ende anfassend, das andere nach oben wenden, ohne da $\beta$ das Gefäß dabei einknickt. Häufig sieht man in diesem Stadium eine starke Schlängelung in situ, namentlich bei kleineren Arterien. Diese ist in ähnlicher Weise, wie die partielle Erweiterung der Gefäße, zu erklären durch die Verkalkung in erschlafttem Zustand; bei der Schlängelung kommen dabei die längsverlaufenden Muskelelemente, bei der Erweiterung die zirkulären in Betracht. An solchen Gefäßen ist in der Regel eine diffuse oder nur Abschnitte der Circumferenz einnehmende Intimawucherung vorhanden, doch kommt es selbst in den hochgradigsten Fällen im Gegensatz zur Arteriosklerose nicht zu degenerativen Prozessen in den Intimawucherungen. Es sind aber auch unter meinem Material einzelne Fälle, in denen selbst in diesem Stadium eine Intimawucherung ausgeblieben ist. In der verkalkton Media sieht man häufig Resorptionsvorgänge, wie ich sie bei der Knochenbildung in arteriosklerotischen Kalkherden ${ }^{8}$ geschildert habe; in zwei Fällen sah ich selbst Knochenbildung bei reiner Mediaverkalkung. Außerdem finden sich die von $\mathrm{Cohn}{ }^{9}$ zuerst beschriebenen Frakturen im Kalk und um die Bruchenden callusartige bindegewebige Wucherungen. Auffallend selten sah ich Thrombusbildung, obwohl Ka ufmann ${ }^{10}$ dieses Vorkommnis als häufige Folge der Mediaverkalkung hinstellt.

$\mathrm{Ob}$ die sekundäre Intimawucherung bei der reinen Media- 
verkalkung bis zur Obliteration des Gefäßes führen kann, vermag ich nicht za beweisen; doch machen einige meiner Fälle es wahrscheinlich. Ich fand mehrmals eine nach abwärts stark zunehmende Mediaverkalkung der Femoralis und dabei eine Obliteration einer Tibialis verbunden mit hochgradiger Mediaverkalkung. Man könnte sich denken, daß in diesen Fällen die Mediaverkalkung eine aufsteigende Richtung angenommen hat, d. h., daß in dem Befund an der Tibialis das vorgeschrittenste Stadium des. Prozesses zu suchen ist. Andererseits kommen aber auch endarteriitische Prozesse an kleinen Arterien vor, die sich erst sekundär durch Mediaverkalkung komplizieren.

\section{Vorkommmen, Ausdehnung und Folgezustände der reinen Mediaverkalkung.}

Wie anfangs erwähnt, kommt die Mediaverkalkung peripherischer Arterien nach den Autoren in der Regel als Alterserscheinung vor; doch gibt unter anderen Marchand (a. a. 0.) das gelegentliche Vorkommen auch bei jugendlicheren Individuen an. Dabei sind scharf zu trennen von der Mediaverkalkung die als Kalkmetastasen bezeichneten Prozesse, die eventuell (wie in dem von Küttuer ${ }^{11}$ beschriebenen Fall) zu ähnlichen klinischen Symptomen führen können und bei denen das makroskopische Verhalten der Arterien an die Mediaverkalkung erinnern kann. Mikroskopisch läßt sich der Unterschied leicht feststellen, da es sich bei den von Kalkmetastasen befallenen peripherischen Arterien um Inkrustationen der im übrigen intakten Intima bei völlig normaler Media und Adventitia handelt.

Unter meinen 55 Fällen von reiner Mediaverkalkung finden sich 4 Fälle präseniler Kalkablagerung, wenn man das beginnende Alter vom vollendeten 50. Jahre ab rechnet. Diese 4 Fälle betreffen nur Männer, wie denn überhaupt das weibliche Geschlecht später und dann in geringerer Ausdehnung (nicht aber immer in geringerem Grade!) von der Mediaverkalkung befallen zu werden scheint. Zwei dieser Männer hatten das 40. Lebensjabr noch nicht erreicht (der eine zählte 35, der andere 36 Jahre), während die beiden anderen $z$ wischen 40 und 50 Jahren standen. Alle übrigen 51 Fälle waren über 50 Jahre alt. Aus der Tabelle I ist $\mathrm{zu}$ ersehen, wie sie sich nach dem Geschlecht auf die ein- 
zelnen Altersklassen verteilen, und in welcher Ausdehnung das Gefäßsystem ergriffen ist.

Tabelle I.

\begin{tabular}{|c|c|c|c|c|c|c|c|c|c|c|c|c|c|}
\hline \multirow[b]{2}{*}{ Ausdehnung } & \multirow[b]{2}{*}{ Zahl } & \multicolumn{12}{|c|}{ Alter } \\
\hline & & Unt & & $\begin{array}{c}40 \\
\mathbf{m}\end{array}$ & & & $\begin{array}{c}-60 \\
w\end{array}$ & $\begin{array}{c}60- \\
\text { m }\end{array}$ & $\begin{array}{c}-70 \\
w\end{array}$ & $\begin{array}{l}70 \\
\mathrm{~m}\end{array}$ & $\begin{array}{c}-80 \\
w\end{array}$ & $\mid \begin{array}{c}\text { Übe } \\
m\end{array}$ & $\begin{array}{c}r 80 \\
w\end{array}$ \\
\hline $\begin{array}{l}\text { 1. Femorales, } \\
\text { Tibiales, Brachi- } \\
\text { ales, Radiales: }\end{array}$ & 3 & - & - & - & - & 2 & - & 1 & - & - & - & - & 一 \\
\hline $\begin{array}{l}\text { 2. Femorales, } \\
\text { Tibiales, Radi- } \\
\text { ales. }\end{array}$ & 10 & - & - & - & - & 1 & - & 4 & - & 2 & 1 & 1 & 1 \\
\hline $\begin{array}{l}\text { 3. Femorales, } \\
\text { Tibiales. }\end{array}$ & 14 & 2 & - & 1 & - & 2 & - & 1 & 1 & 1 & 2 & 2 & 1 \\
\hline 4. Femorales. & 15 & - & - & 1 & - & 1 & 3 & 1 & 4 & 3 & 2 & 1 & - \\
\hline $\begin{array}{l}\text { 5. Tibiales, } \\
\text { Radiales. }\end{array}$ & 4 & - & - & - & - & - & - & 1 & - & 2 & - & - & 1 \\
\hline 6. Tibiales. & 5 & - & 一 & - & - & 1 & - & - & 1 & - & 1 & - & 2 \\
\hline 7. Radiales. & 4 & - & - & - & - & 1 & - & 2 & - & - & 1 & - & - \\
\hline Summa & 55 & 2 & - & 2 & - & 8 & 3 & 10 & 6 & 8 & 7 & 4 & 5 \\
\hline
\end{tabular}

Die Femoralis ist das Gefä $B$, dessen Media am öftesten Kalksalze aufzunehmen scheint. Unter meinen Fällen ist sie im ganzen 24 mal erkrankt, davon $15 \mathrm{mal}$ ohne Beteiligung anderer peripherischer Gefäße. Bei diesen isolierten FemoralisMediaverkalkungen findet man in der Regel den Prozeß links und rechts gleichweit vorgeschritten. Während meist der oberste Teil unterhalb des Ligamentum Poupartii frei ist, setzt in dem darauffolgenden Abschnitt die makroskopisch deutlich sichtbare 
Verkalkung mit rasch nach der Peripherie zu steigender Intensität ein, um sich in der Kniekehle oder bald nach der Teilung in die beiden Hauptendäste allmählich wieder zu verlieren. Doch nimmt die Verkalkung in diesen Fällen niemals höhere Grade an. Man sieht meist zentralwärts die oben beschriebenen gelblichen Fettspangen und -streifen, die schnell nach der Peripherie hin in Kalkstreifen übergehen. Dabei ist die äußere und innere Oberfläche des Gefäßes noch relativ glatt und die bekannte gänsetracheaartige Beschaffenheit kommt nicht zum Ausdruck.

Anders verhalten sich kleinere Arterien, wenn sie allein vom VerkalkungsprozeB ergriffen sind. Bei den 4 Fällen von isolierter Radialisverkalkung in meinem Material war einmal nur in der rechten Radialis eine herdweise Verkalkung vorhanden, die erst nach dem Aufschneiden des Gefäßes in Form kleiner Plättchen mit eingesunkener, aber glatter Intimabedeckung zu sehen war. Ein anderes Mal waren rechts und links ähnliche, aber über weitere Strecken der Gefäße scheinbar unregelmäßig zerstreute Kalkherde zu beobachten. In den beiden letzten Fällen sah man richtige Kalkringe, die von oberhalb des Handgelenks in ziemlich gleichmäßiger Intensität bis zum Übergang in die Brachialis hinauf reichten und dort schnell aufhörten.

Die 5 Fälle mit alleiniger Tibialisverkalkung betreffen Individuen, die an hochgradiger Arteriosklerose der Femorales litten. Hier hatte sich zu den sklerotischen Prozeßen eine Mediaverkalkung hinzugesellt, und diese setzte sich nach Aufhören der endarteriitischen Erscheinungen kontinuierlich in die Tibiales fort. Ähnlich liegen die 4 Fälle mit isolierter Radialis und Tibialisverkalkung für die letztere, während sich in der Radialis dabei einmal eine herdförmige Kalkablagerung und 3 mal richtige Kalkringe fanden.

Sehr viel schwerere Erkrankungen stellen die Fälle dar, in denen die Hauptgefäße der unteren Extremitäten oder die der unteren und oberen befallen waren. Dabei hat man sich aber nicht vorzustellen, daß je größer die Ausdehnung, desto höhere Stadien des Verkalkungsprozeßes zu finden wären. Im Gegenteil: die Fälle, bei denen die Gefässe der unteren Extremität in starre Rohre verwandelt waren, liessen eine Verkalkung der Armarterien vermissen, und bei der allgemeinen Verkalkung der 
Extremitätengefässe war nur das Stadium der Kalkringe zu konstatieren. - Wie aus der Tabelle I ersichtlich, ist wieder die untere Extremität bei weitem am häufigsten ergriffen; dagegen konnte ich keine isolierten Verkalkungen der Gefässe des ganzen Armes finden; nur in den 3 Fällen allgemeiner peripherischer Mediaverkalkung war die Arteria brachialis in Mitleidenschaft gezogen worden.

Daraus, daß isolierte Femoraliserkrankungen stets geringere Grade der Verkalkung zeigen, als wenn die gesamten Beinarterien betroffen sind, darf nicht geschlossen werden, daß der Prozeß immer in der Femoralis beginst und auf die Tibiales sich fortsetzt; denn häufig trifft man bei Verkalkung von Femoralis und Tibialis in letzterer sehr viel vorgeschrittenere Stadien an. Dagegen scheint die Mediaverkalkung an den Armarterien stets eine aufsteigende Richtung zu nehmen. Dabei ist der Prozeß an beiden Extremitäten in den höheren Graden der Erkrankung stets ein kontinuierlicher; niemals habe ich eine Unterbrechung gesehen in der Weise etwa, daß die Femoralis verkalkt, die Poplitea frei und die Tibiales wiederum mit Kalksalzen imprägniert gewesen wären.

Die Aufstellung von Folgezuständen der Mediaverkalkung ist mit großer Vorsicht vorzunehmen, da selten die Fälle so rein sind, da $\beta$ für etwaige Befunde nur die Mediaerkrankung in Betracht gezogen werden könnte. Da, wie ich weiter unten ausführen werde, die reine Calcificatio tunicae mediae peripherischer Arterien häufig eine Arteriosklerose centraler Gefässe begleitet, ist es bei der Obduktion äusserst schwer zu beurteilen, was als Folge der einen anzusehen ist, und welche Veränderungen im Organismus die andere gezeitigt hat. Wichtig sind daher vor allen Dingen die Fälle, wo sich keine oder nur eine verschwindend geringe anderweitige Gefäßerkrankung neben der Mediaverkalkung nachweisen lässt, und von diesen Fällen finden sich unter meinem Material 12 (s. unten Fall 1, 2, 4, 7, 8, 14, $15,28,29,31,32,33)$. Obwohl unter diesen 12 Fällen einige ganz besonders schwer sind (Fall 14, 15, 31), andere die peripherischen Arterien in weiter Ausdehnung verkalkt zeigen (Fall 1, $2,4,7,8$ ), war doch in keinem Falle eine in die Augen fallende Hypertrophie des linken Ventrikels za konstatieren. Ob hierbei 
der Umstand die Rolle spielt, daß trotz des Elastizitätsverlustes der peripherischen Gefässwände keine oder doch nur eine unwesentliche Beeinträchtigung der Querschnittssumme aller Gefäßlumina eingetreten ist, muß ich dahingestellt sein lassen. Zur Entscheidung dieser Frage müßten ausgedehntere Kenntnisse der pathologischen Gefäßphysiologie vorhanden sein, und man müßte über eingehende klinische Mitteilungen über die Tätigkeit der Patienten verfügen, um festzustellen, ob überhaupt und inwieweit die Extremitäten in ihrer Nahrungszufuhr durch die Verkalkung beeinträchtigt worden waren.

Der relativ häufige Befund von Endophlebitis und Thrombose der Begleitvenen bei Mediaverkalkung machen, wie Orth bereits hervorgehoben hat, eine verlangsamte, bezw. verminderte Blutzufuhr durch die erkrankten Arterien allerdings wahrscheinlich. Aber ob diese Venenbefunde erst sekundär entstehen, oder ob dieselben Ursachen für sie, wie für die degenerativen Prozeße in den Arterien bestanden haben, dafür giebt mein Material keine Anhaltspunkte, da, wie bereits oben erwähnt, die Venenerkrankung ganz inkonstant gefunden wurde. Faßt man die Thrombose der Vene als Folge der Mediaverkalkung auf, so läßt sich in 3 meiner Fälle der Tod der Patienten indirekt auf letztere zurückzuführen: $3 \mathrm{mal}$ war eine Lungenembolie durch die Thrombose der Vena femoralis bedingt. In einem weiteren Falle hatte die Thrombose derselben Vene mit ihren Hautästen eine Geschwärsbildung am Unterschenkel bewirkt. - Nimmt man ferner an, daß die sekundäre Intimawucherung bei Mediaverkalkung allmählich bis zur Obliteration des betreffenden Gefäßes führen kann, so finden weitere 3 Fälle in der Mediaverkalkung ihre Erklärung, bei denen es sich um senile Gangrän der Füße bei bestehender Mediaverkalkung der Femorales und obliterierender Intimawucherung mit verkalkter Media der Tibiales handelte. Doch läßt sich irgend etwas Abschließendes, wie schon vorher bemerkt, hierüber vorläufig nicht sagen. In allen anderen Fällen lag an der Leiche nichts vor, was in Zusammenhang mit der Mediaverkalkung hätte gebracht werden können.

Bei Beantwortung der Frage, welche Lebensbedingungen als Ätiologie der degenerativen und zur Ablagerung von Kalksalzen 
führenden ProzeBe in der Gefässwand in Betracht zu ziehen sind, müssen vor allen Dingen wieder die nicht durch Arteriosklerose anderer Gefäße komplizierten Fälle berücksichtigt werden, da diese am ehesten einen ätiologischen Unterschied zwischen Mediaverkalkung und Arteriosklerose aufweisen. Die vorher erwähnten 12 Fälle in meinem Material geben nun in ihren auf den Stationen des hiesigen Krankenhauses erhobenen Anamnesen durchaus keine einheitlichen Anhaltspunkte, die mit der Mediaverkalkung in Zusammenhang gebracht werden könnten. Dabei ist zu berücksichtigen, daß fast durchweg bei Aufstellung der Anamnese nur die auf das Grundleiden der Patienten bezüglichen Angaben aufgeschrieben wurden. - Nach dem Geschlecht teilen sich diese Fälle in 10 männliche und 2 weibliche; das Alter schwankt zwischen 47 und 79 Jahren, ist also dem gleich, bei dem man auch arteriosklerotische Gefäßveränderungen anzutreffen pflegt. Der Beruf ist ein wechselnder: neben Arbeitern finden sich Kommis und neben Seeleuten Handwerker. Überstandene Geschlechtskrankheiten und Unmäßigkeit im Trinken sind nur in je 2 Fällen in der Anamnese angegeben. In weiteren 4 Fällen haben die Patienten vor längerer oder kürzerer Zeit Wechselfieber, Pocken, Dysenterie und Cholera überstanden. Von chronischen Knochenleiden, die bekanntlich nach erfolgter Resorption von Kalk zu Kalkablagerungen an anormalen Stellen des Organismus führen, ist nirgends die Rede.

Bis auf die Prävalenz des männlichen Geschlechts sind also die Bedingungen, unter denen es zur Mediaverkalkung kommt, vorläufig völlig dunkel. Dagegen finden sich Anhaltspunkte für die in den Lehrbüchern festgelegte Ansicht, daß die degenerativen, der Kalkablagerung vorausgehenden Prozeße eine mangelhafte Ernährung der Gefäßwand zur Ätiologie haben. Da die Verkalkung, wie hervorgehoben, meist größere Abschnitte des Gefäbsystems einnimmt, so ist es von vornherein wahrscheinlich, daß dabei nicht lokale Ernährungsstörungen, sondern wohl meist Herabsetzungen der Gesamternährung des Organismus in Betracht kommen. Krankheiten, die solche Zustände herbeiführen können, finden sich nun in den sämtlichen in Frage stehenden Fällen: 4 mal bestanden schwere Krebskachexien, 4 mal waren durch chronische Lungenleiden (Tuberkulose und Emphysem) die Be- 
dingungen zur Herabsetzung der Gesamternährung gegeben, und in den letzten 4 Fällen können einmal eine sogen. Tropenkachexie, 2 mal chronische Leiden des Harnapparates (Prostatahypertrophie mit Folgezuständen) und einmal ein schwerer Aortenfehler als ätiologische Momente herangezogen werden.

\section{Mediaverkalkung und Arteriosklerose.}

Wie ich in der Einleitung schon bemerkte, scheint der Schluss von der klinisch leicht za konstatierenden Mediaverkalkung peripherischer Arterien auf eine Sklerose der Aorta oder anderer Gefäßgebiete in manchen Fällen seine Berechtigung zu haben. Aber ebenso sicher kommen Fälle vor, bei denen sich eine Mediaverkalkung in geringerer oder größerer Ausdehnung findet, ohne dass man auch nur eine Spur von arteriosklerotischen Veränderungen im Organismus antrifft. Es gilt also festzustellen, in welcher Häufigkeit die beiden Erkrankungen sich kombinieren und $o b$ aus der Schwere der einen ein Schluss auf den Grad der anderen gezogen werden darf. Dabei bin ich mir selbstverständlich bewußt, dass die fühlbare Verkalkung peripherischer Arterien nur ein Anhaltspunkt für die klinische Diagnose auf Arteriosklerose ist, und da.B man noch über verschiedene andere Symptome verfügt, deren Erörterung hier nicht am Platze ist.

Meine 55 Fälle von Mediaverkalkung peripherischer Arterien verhielten sich folgendermaßen:

I. Verkalkung der Art. femorales, tibiales, brachiales, radiales.

1. $\sigma^{7} 64$ Jahre. In der Aorta am Abgange der Intercostalarterien geringe weissgelbe Platten. Hochgradige Mediaverkalkung peripherisch.

2. $ð 56$ Jahre. Am Bogen der Aorta ein kleiner Herd wit Kalk Sonst völlig frei. - Peripherisch Mediaverkalkung mittleren Grades.

3. $ð 58$ Jahre. Aorta wegen Überführung nicht untersucht. - Peripherisch hochgradige Mediaverkalkung.

II. Verkalkung der Art. femorales, tibiales, radiales.

4. $\sigma^{7} 58$ Jahre. In der Aorta vereinzelte Platten am Abgang der Intercostalarterien. Peripherisch Mediaverkalkung hohen Grades.

5. $0^{\pi} 67$ Jahre. In der Aorta einzelne weissgelbe Herde ohne regressive Metamorphosen. Peripherisch Mediaverkalkung hohen Grades.

6. ఫิ 62 Jabre. In der Aorta geringe nodöse Sklerose. Art. coronariae hochgradig sklerotisch. Peripberisch starke Mediaverkalkung.

7. $\sigma^{7} 72$ Jahre. An der unteren Wand des Aortenbogens ein Herd 
mit Kalkplatte. Im Brustteil der Aorta eine weitere Platte, milchigweiss, nodös. - Peripherisch Mediaverkalkung mittleren Grades.

8. J 60 Jahre. Im Aortenbogen eine Platte ohne regressive Metamorphose. Peripherisch Mediaverkalkung mittleren Grades.

9. o 62 Jahre. In der Aorta nodöse Sklerose, Platten teils atheromatös, teils mit Kalkeinlagerungen, teils fibrös. - Peripherisch Mediaverkalkung mittleren Grades.

10. of $^{7} 1$ Jahre. Aortenklappen stark sklerotisch, mit Kalk (Insufficienz). Im ganzen Verlauf der Aorta nodöse Sklerose mit Atherom. Art. coronariae und basilares sklerotisch. Peripherisch hochgradige Verkalkung der Media.

11. 774 Jabre. In der Aorta fleckweise Sklerose mit Atherom und Kalk. - Peripherisch hochgradige Mediaverkalkung.

12. ㅇ 76 Jahre. In der ganzen Aorta diffuse Sklerose mit Kalk, Geschwüren und Thromben. Fortsetzung in die Iliacae. Coronariae sklerotisch. - Peripherisch geringe Mediaverkalkung.

13. 오 81 Jahre. Im Anfangsteil der Aorta mäßige, im weiteren Verlauf stark zunehmende Sklerose, diffus mit Kalk. In der Abdominalis ein langer, wandständiger Thrombus. - Basilares und Coronariae sklerotisch, ebenso die Nierenarterien. - Peripherisch starke Mediaverkalkung.

\section{Verkalkung der Art. femorales und tibiales.}

14. $\sigma^{\pi} 52$ Jabre. In der Aorta flache, gelbweiße Platten am Abgang der Intercostalarterien. - Peripherisch Mediaverkalkung bohen Grades.

15. \& 63 Jahre. In der Aorta einige flache, gelbliche Platten, sonst glatt und normal. Peripherisch Mediaverkalkung höchsten Grades.

16. $\sigma^{7} 65$ Jahre. In der Aorta vereinzelte erhabene bindegewebige Herde. - An den Beinen nach unten zu stark zunebmende Mediaverkalkung.

17. đ 53 Jahre. Anfangsteil, Bogen und Brustteil voll sklerotischer Plaques, die sich auch auf die Äste fortsetzen. In der Abdominalis Abnahme, in den Iliacae einige atheromatöse Herde. Nirgends Kalk. - Coronariae nur am Anfang sklerotisch. - Peripheriseb Mediaverkalkung mittleren Grades.

18. $0^{\pi} 45$ Jahre. In der Aorta von den Klappen bis zur Teilungdiffuse Sklerose mit Atherom, ohne Geschwüre und Kalk. In der Iliaca com. sin. eine sklerotische Platte. - Peripherisch Mediaverkalkung hohen Grades.

19. $\sigma^{7} 36$ Jahre. Direkt über den Aortenklappen scharf umschriebene, erhabene Sklerose mit Atherom. In der ganzen übrigen Aorta einzelne fibröse Herde. - Peripherisch Mediaverkalkung mittleren Grades.

20. $\sigma^{\tau} 35$ Jahre. Dicht über den Aortenklappen scbarf umschriebene, erhabene Plaques, die die Coronarostien ganz, bezw. fast gavz verlegen. Im Bogen und der Aorta descendens einzelne flache fibröse Herde. - Coronariae sklerotisch. - Peripherisch geringe Mediaverkalkung. 
21. ㅇ 75 Jahre. In der Aorta mäßige diffuse Sklerose mit einzelnen erhabenen Platten mit Kalk. Iliacae com. im Anfang sklerotisch. Basilaris bochgradig sklerotisch. - Mediaverkalkung geringen Grades.

22. ㅇ 82 Jahre. In der Aorta einzelne Platten, z. T. verfettet, z. T. atheromatös. An der Teilungsstelle etwas Kalk. Iliacae mäßig sklerotisch. Mediaverkalkung hohen Grades.

23. 오 70 Jabre. Im Aortenbogen starke diffuse Sklerose mit Kalk und Atherom. Weiter nach unten Abnalıme, bis Iliacae völlig frei. Mediaverkalkung geringen Grades.

24. O $^{83}$ Jahre. Aorta ascendens, Bogen und Brustaorta geringe nodöse Sklerose, die in der Baucbaorta stark zunimmt; Coronarjae sklerorotisch. - Peripherisch Mediaverkalkung mäßigen Grades.

25. Of 84 Jahre. Im ganzen Verlauf der Aorta grosse flache Platten mit Blutungen, Thromben und grossen Kalkplatten. - Mediaverkalkung höchsten Grades.

26. O $^{75}$ Jahre. In der ganzen Aorta hochgradige diffuse Sklerose mit Atherom, Kalk, Geschwüren, Blutungen und Thromben. Fortsetzung der Sklerose auf die Ȧste und die Iliacae. - Peripherisehe Mediaverkalkung höchsten Grades.

27. Of 79 Jahre. Aorta mit nach unten zunehmender, erst fleckiger, dann mehr und mehr konfluierender Sklerose. An der Teilungsstelle große Kalkplatten. Iliacae sklerotisch, ebenso die Bauchẳste der Aorta. - Mediaverkalkung hohen Grades.

IV. Isolierte Verkalkung der Femoralis.

28. O 47 Jahre. In der Aorta einzelue flache fibröse Platten. Mediaverkalkung der Femoralis geringen Grades.

29. O $^{7} 5$ Jahre. Am Bogen der Aorta geringe Platten von weifgelber Farbe. Basilaris sklerotisch. - Verkalkung mittleren Grades der Femoralis.

30. 우 67 Jahre. Am Bogen und im Brustteil der Aorta einzelne sklerotische Herde. - In der Femoralis beginnende Mediaverkalkung.

31. $0^{7} 65$ Jahre. Am Abgang der Intercostalarterien einzelne gelb. weile Platten. - In der Femoralis und ibren Seitenästen Mediaverkalkung hoben Grades.

32. 万79 Jabre. An der unteren Wand des Bogens der Aorta ein sklerotischer Berd, sonst völlig frei. - In der Femoralis Mediaverialkung mittleren Grades.

33. ㅇ 69 Jabre. In der Aorta einzelne flache, gelbweiße Platten. Beginnende Mediaverkalkung der Femoralis.

34. 우 61 Jabre. Aorta mit einzelnen flachen sklerotischen Herden. - Femorales mit Mediaverkalkung mäßigen Grades.

35. 73 Jahre. In der Aorta atheromatöse Herde mit Kalkablagerungen. - Femorales mit Mediaverkalkung hohen Grades. 
36. ð 83 Jahre. Diffuse Sklerose mäßigen Grades der Aorta. Hochgradige Mediaverkalkung der Femorales.

37. +69 Jabre. In der Aorta hochgradige fleckweise Sklerose mit kleinen Kalkplatten, die nach unten hin stark abnimmt. - Mediaverkalkung mittleren Grades der Femorales.

38. 우 56 Jahre. Vom Anfangsteil bis zur Teilung der Aorta starke herdförmige Sklerose mit Geschwüren und Kalkplatten. Ringstenose der Coronarostien. Coronararterien mit einzelnen sklerösen Platten. - Beginnende Mediaverkalkung der Femorales.

39. 오 56 Jahre. Anfangsteil frei. Brust- und Bauchteil der Aorta mit hochgradiger Sklerose, die sich fortsetzt auf die Äste. Iliacae, Coronariae, Basilares sklerotisch. - Mediaverkalkung mittleren Grades der Femorales.

40. 오 58 Jahre. Anfangsteil und Bogen der Aorta mit hochgradiger, diffuser Sklerose, grossen Kalkplatten und Wandthromben. In der Aorta descendens einzelne Platten am Abgang der Intercostalarterien. - In den Femorales hochgradige Mediaverkalkung.

41. $\sigma^{7} 74$ Jahre. In der ganzen Aorta hochgradige Sklerose mit Blutungen, Geschwüren und Kalk. - Mediaverkalkung mittleren Grades der Femorales.

42. \& 76 Jabre. Die ganze Aorta stark diffus sklerotisch mit großen Kalkplatten und Geschwüren. Iliacae desgl. - Hochgradige Mediaverkalkung der Femorales.

V. Isolierte Verkalkungen der Tibiales und Radiales.

43. o 64 Jahre. Anfangsteil frei. Im weiteren Verlauf der Aorta hochgradige Sklerose, die sich fortsetzt auf die Äste, die Iliacae und Femorales. Coronar- und Basilararterien sklerotisch. - In der r. Radialis ein kleiner Mediakalkherd, Tibiales gänsetracheaartig verkalkt.

44. 9 85 Jahre. In der Aorta descendens hochgradige Sklerose mit Kalkplatten. In den Halsgefässen einzelne Platten mit Kalk. Femorales neben Plaques Mediaverkalkung. - Tibiales und Radiales tnit reiner Mediaverkalkung.

45. ఫै 77 Jahre. In der ganzen Aorta böchstgradige Sklerose mit Geschwüren und Kalkplatten. Anfangsteil ofenrohrartig starr erweitert. Iliacae und Femorales mit sklerotischen Platten. - Tibiales und Radiales gänsetracheaartig verkalkt.

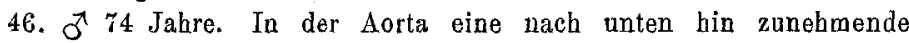
diffuse Sklerose, an der Teilungsstelle grosse Kalkplatten. Art. coron., basil., iliac., femor. sklerotisch. - Tibiales starr verkalkt. In den Radiales einzelne Mediakalkherde.

47. $\sigma^{7} 58$ Jahre. In der Aorta Atheromatose. Femorales mit einzelnen Platten. - Mediaverkalkung der Tibiales.

48. ㅇ 79 Jahre. Aorta ascendens cylindrisch erweitert. In ihr und der Aorta thoracica nodöse Sklerose mittleren Grades. Bauchaorta mit 
hochgradiger, mehr diffuser Sklerose, Geschwüren und Kalkplatten; Fortsetzung auf die Äste, die Iliacae und Femorales. - Tibiales mit reiner Mediaverkalkung.

49. +82 Jahre. Im ganzen Verlauf der Aorta starke diffuse Sklerose, flache Platten mit Kalkplatten und klennen wandständigen Thromben. Iliacae und Femorales fleckweise Sklerose; in letzteren daneben Mediaverkalkung. - Tibiales za starren Rohren verkalkt.

50. 우 60 Jahre. Am Bogen der Aorta aneurysmatische Erweiterung. Im ganzen Verlauf starke diffuse Sklerose. Ebenso in den Iliacis und den Ästen der Aorta. In den Femorales neben Platten Mediaverkalkung. In den Tibiales reine Mediaverkalkung.

51. 우 82 Jahre. In der Aorta vereinzelte hochgradige Herde neben allgemeiner Sklerose mittleren Grades. Äste der Aorta hochgradig sklerotisch. Femorales mit alten organisierten Thromben. - Hochgradige Nediaverkalkung der Tibiales.

52. O 50 Jahre. In der Aorta Atherom mit Kalk. - Radiales mit Mediaverkalkung mittieren Grades.

53. 73 Jahre. In der ganzen Aorta nodöse Sklerose mit Atherom, ebenso in den Iliacis und den Arterien der Beine. - In der l. Radialis ein Herd von Mediaverkalkung.

54. Of 61 Jahre. Starke Sklerose der Aorta mit Kalkplatten. In den Femorales neben Platten Mediaverkalkung. Coronar- und Basilararterien sklerotisch. - Radiales gänsetracheaartig verkalkt.

55. $\sigma^{7} 60$ Jahre. Aneurysma eines Sinus Valsalvae. Grosses sackförmiges Aneurysma am Anfang des Brustteils mit starker Wandthrombose. Im ganzen Verlauf der Aorta starke diffuse Sklerose mit viel Kalk. Bogenäste, Iliacae und Beinarterien sklerotisch. - In beiden Radiales einzelne Mediakalkherde.

Nach dem Beispiele anderer Autoren teile ich die arteriosklerotischen Prozeße in folgende drei Grade ein: 1. Stadium: bindegewebig-elastische Platten von weißgelber Farbe; 2. Stadium: Atherom; 3. Stadium: Geschwürsbildung, Kalkablagerung, Thrombierung u. s. w. - Folgt man diesem Einteilungsprinzip, so ergiebt sich für meine Fälle die Aufstellung in Tabelle II.

Dabei sind zu der Arteriosklerose ersten Grades die Fälle zugezählt, bei denen nur an der untern Wand des Aortenbogens ein Herd gefunden wurde (Fall 2, 7, 8, 29, 32). Diesen Herd trifft man aber in vorgeschrittenem Alter bei allen Leichen an, so daß man nicht fehl geht, wenn man ihn als physiologische Alterserscheinung auffaßt und die betreffenden Fälle als frei von Arteriosklerose bezeichnet. 
Aus der Tabelle II geht nun hervor, daß in den Fällen von weit ausgedehnter Mediaverkalkung meist nur geringe Grade von Arteriosklerose an der Aorta gefunden wurden. Dabei ist in Betracht zu ziehen, daß diese Fälle, wie schon oben bemerkt, nicht die höchsten Grade der Mediaverkalkung darzustellen

Tabelle II.

\begin{tabular}{|c|c|c|c|c|}
\hline Mediaverkalkung in: & 1. Grades & $\begin{array}{l}\text { rteriosklero } \\
\text { 2. Grades }\end{array}$ & 3. Grades & Zahl \\
\hline $\begin{array}{l}\text { 1. Femorales, } \\
\text { Tibiales, } \\
\text { Bracbiales, } \\
\text { Radiales }\end{array}$ & 3 & 一 & - & 3 \\
\hline $\begin{array}{l}\text { 2. Femorales, } \\
\text { Tibiales, } \\
\text { Radiales }\end{array}$ & 5 & 3 & 2 & 10 \\
\hline $\begin{array}{l}\text { 3. Femorales, } \\
\text { Tibiales }\end{array}$ & 3 & 5 & 6 & 14 \\
\hline 4. Femorales & 7 & 3 & 5 & 15 \\
\hline $\begin{array}{l}\text { 5. Tibiales, } \\
\text { Radiales }\end{array}$ & - & - & 4 & 4 \\
\hline 6. Tibiales & 一 & 1 & 4 & 5 \\
\hline 7. Radiales & 一 & 2 & 2 & 4 \\
\hline Summa & 18 & 14 & 23 & 55 \\
\hline
\end{tabular}

brauchen. Aber es finden sich doch unter den 8 Fällen, wo bei Arteriosklerose 1. Grades die Arterien der obern und untern Extremität in mehr oder weniger weiter Ausdehnung verkalkt waren, 4 Fälle von höchstgradiger Mediaverkalkung, d. h. die Arterien waren da streckenweise in starre Kalkrohre verwandelt. Andererseits sind aber auch nicht die hochgradigen Arteriosklerosen der Aorta durchweg mit hohen Stadien der Mediaverkalkung peripherischer Gefässe kombiniert. Daher ist weder der SchluB von weitausgedehnter Mediaverkalkung auf Arteriosklerose überhaupt, noch der von hohen Graden

Virehows Archiv f. pathol. Anat. Bd. 171. Hft. 1. 
der Mediaverkalkung auf vorgeschrittene Stadien der Arteriosklerose ohne weiteres berechtigt. Die beiden Prozeße können im Organismus nebeneinander hergehen, ohne anscheinend auch nur im geringsten voneinander beeinflußt zu werden. Nimmt man sich z. B. aus meinem Material die 21 Fälle heraus, bei denen die Radiales in den Verkalkungsprozess einbezogen waren, so ergibt sich, daß diese Arterien $15 \mathrm{mal}$ in höheren Graden verkalkt waren, während 6 mal nur kleine Kalkplättchen, die wahrscheinlich der klinischen Untersuchung entgangen wären, gefunden wurden. Wollte man aus den 15 Fällen hochgradiger Verkalkung auf starke Arteriosklerose der Aorta schließen, so würde man in 10 Malen fehl gehen. Andererseits finden sich unter den Fällen mit kleinen Kalkplättchen in den Radiales 4 Fälle hochgradiger Arteriosklerose der Aorta. Es lassen sich also keine Regeln aufstellen für die Befunde, und nur das ist hervorzuheben, da $B$ der klinische Befund verkalkter peripherischer Arterien durchaus nicht ohneweiteres zu der Diagnose auf Arteriosklerose der Aorta berechtigt.

Bei der Sektion der Fälle von mehr oder weniger starker Arteriosklerose der Aorta und ihrer Äste, kombiniert mit Mediaverkalkung peripherischer Arterien, findet man in der Regel zwischen beiden Erkrankungsgebieten Gefäßabschnitte, die völlig normal erscheinen. So besitze ich mehrere Präparate z. B., in denen die Aorta mit ihren Bogenästen, die Iliacae communes und externae Arteriosklerose zeigen, während die oberen Abschnitte der Femorales und Brachiales vollständig frei sind und die darauf peripheriewärts folgenden Gefäßbezirke eine exquisite reine Mediaverkalkung darbieten. In anderen Fällen sind weite Strecken normaler Gefäßwand zwischen den beiden Erkrankungsgebieten. Es kommt aber auch vor, und das habe ich hauptsächlich an der unteren Extremität beobachtet, daß Gefäßabschnitte Arteriosklerose und Mediaverkalkung nebeneinander zeigen und daß erstere peripheriewärts allmählich ganz verschwindet, während die Mediaverkalkung bestehen bleibt und meist noch zunimmt. So erklären sich die Fälle aus der Tabelle II mit isolierter Tibialisverkalkung bei Arteriosklerose 3. Grades. Bei diesen dehnte sich die schwere Arteriosklerose 
bis weit in die Femorales hinein aus; diese Arterien zeigten von der Mitte ihres Verlaufs etwa an eine nach unten zunehmende Mediaverkalkung, die nach Aufhören der sklerotischen Prozesse ohne Unterbrechung in die Tibiales sich fortsetzte. Dagegen war bei den 2 Fällen isolierter Radialisverkalkung mit hochgradiger Arteriosklerose nichts von derartigen Übergängen zu konstatieren.

Es ist von vornherein nicht wahrscheinlich, daß in allen Fällen, bei denen klinisch auf Arteriosklerose aus dem "Atherom" (Strümpell) der fühlbaren Arterien diagnostiziert wurde, eine Mediaverkalkung der Extremitätengefäße vorliegt; vielmehr muß man annehmen, daß eine wirkliche Arteriosklerose peripherischer Arterien unter Umständen (z. B. bei Verkalkung atheromatöser Herde) dieselben klinischen Erscheinungen macht. Es bleibt also noch übrig festzustellen, wie sich die Häufigkeit dieser beiden Erkrankungen zueinander verhält, und in welcher Form die Arteriosklerose an den Extremitätenarterien aufutreten pflegt. - Unter meinen 55 Fällen reiner Mediaverkalkung sind 12 Fälle, die neben dieser Erkrankung an einzelnen Gefäßen arteriosklerotische Veränderungen an anderen Arterien der Extremitäten zeigen, z. B. in der vorher schon erwähnten Weise, daß die zentrale Sklerose sich auf die Gefäße des Oberschenkels oder des ganzen Beines ausdehnt, während die Radiales Mediaverkalkung zeigen. In weiteren 18 Fällen fand sich dagegen nur Endarteriitis deformans an den peripherischen Arterien, und in den übrigen 57 Fällen waren überhaupt keine Veränderungen der Extremitätengefässe bei bestehender Sklerose innerer Arterien nachzuweisen. Es fand sich also unter 130 Fällen von Gefäßerkrankungen:

43 mal nur Mediaverkalkung

12 mal Mediaverkalkung und Arteriosklerose

$18 \mathrm{mal}$ nur Arteriosklerose

57 mal keinerlei Erkrankung

$\left\{\begin{array}{c}\text { der } \\ \text { Extremitäten- } \\ \text { arterien. }\end{array}\right.$

Daraus geht hervor, daß an den Gefäßen der Arme und Beine die Mediaverkalkung häufiger vorkommt, als die Arteriosklerose. Wenn man sich die Arteria radialis ans diesem Material herausgreift, die ja bei weitem am häufigsten zur Palpation be- 
nutzt wird, so tritt das Häufigkeitsverbältnis beider Gefäßerkrankungen noch sehr viel deutlicher hervor: Unter meinen 130 Fällen fand sich 21 mal eine Mediaverkalkung und nur 3 mal eine Arteriosklerose an den Radiales. Und in diesen 3 Fällen waren nur einzelne kleine Platten ohne Kalkablagerung zu konstatieren, die aller Wahrscheinlichkeit nach der klinischen Palpation entgangen wären. Daher kann man wohl behaupten,

Tabelle III.

\begin{tabular}{|c|c|c|c|c|c|}
\hline Arteriosklerose in: & $\begin{array}{r}\text { Zentral } \\
\text { 1. Grades }\end{array}$ & $\begin{array}{l}\text { le Arterio: } \\
\text { 2. Grades }\end{array}$ & $\begin{array}{l}\text { shlerose } \\
3 . \text { Grades }\end{array}$ & $\begin{array}{c}\text { Zabl } \\
\text { der } \\
\text { Fălle }\end{array}$ & $\begin{array}{l}\text { Kombiniert mit } \\
\text { Mediaverkaikung } \\
\text { in: }\end{array}$ \\
\hline $\begin{array}{l}\text { 1. Femorales, } \\
\text { Tibiales, } \\
\text { Bracbiales, } \\
\text { Radiales }\end{array}$ & - & 1 & - & 1 & - \\
\hline $\begin{array}{l}\text { 2. Femorales, } \\
\text { Tibiales }\end{array}$ & - & 1 & 3 & 4 & Radiales 2 mal \\
\hline 3. Femorales & 2 & 3 & 18 & 23 & $\begin{array}{l}\text { Radiales u. } \\
\text { Tibiales } \\
\text { Tibiales } 5 \mathrm{mal} \\
\text { Radiales } 1 \mathrm{mal}\end{array}$ \\
\hline \multirow[t]{2}{*}{ 4. Radiales } & - & - & 2 & 2 & - \\
\hline & 2 & 5 & 23 & 30 & Im ganzen $12 \mathrm{mal}$ \\
\hline
\end{tabular}

daß in den meisten Fällen, bei denen die Radiales intra vitam als starre, geschlängelte, fragile Rohre zu fühlen sind (Strümpell'sches „Atherom“), keine Arteriosklerose dieser Gefässe, sondern eine Modiaverkalkung vorliegt.

Ähnlich liegen die Verhältnisse auch bei den übrigen Extremitätenarterien. Tritt Arteriosklerose in ihnen auf, so handelt es sich in der ïberwiegenden Mehrzahl um nodöse Prozesse, die Ausläufer einer meist hochgradigen zentralen Sklerose darstellen.

Aus der Tabelle III gehr hervor, daß die untere Extremität ebenso wie von der Mediaverkalkung, auch von der Endarteriitis deformans bevorzugt wird; in der oberen fand ich die letztere überhaupt nur $3 \mathrm{mal}$ im ganzen. Ferner sieht man, daß die peripherische Arteriosklerose meist kombiniert ist mit schwerer 
zentraler Gefäßerkrankung, daß aber auch bei diesen Prozessen Fälle vorkommen, in denen die Ausdehnung auf die Extremitätengefäße nicht den Schluß auf eine hochgradige Erkrankung innerer Arterien gestattet. - Unter den Fällen von Arteriosklerose der Femorales sind manche, wo sich neben den endarteriitischen Prozessen auch deutliche Verkalkung der Media fand; einige von diesen zeigen dabei das vorher schon erwähnte Verhalten, daß nach Aufhören der Arteriosklerose die Mediaverkalkung sich kontinuierlich peripheriewärts fortsetzt in die Tibiales.

$\mathrm{Da}$, wo sich beide Erkrankungen kombinieren, fällt ein Hülfsmittel für die klinische Differentialdiagnose zwischen Mediaverkalkung und Arteriosklerose weg, das in den reinen Fällen große Sicherheit bietet, und auf das E. Fraenkel ${ }^{12}$ und Saenger ${ }^{13}$ schon hingewiesen haben: die Durchleuchtung mit Röntgenstrahlen. Arterien mit Mediaverkalkung selbst nur geringen Grades geben im Röntgenbilde kräftige Schatten, die mehr oder weniger zusammenhängend das Gefäß deutlich sichtbar machen, während Arterien mit Arteriosklerose ohne Kalkablagerung gar nicht oder nur ganz schwach auf Röntgenplatten zu sehen sind. Finden sich in atheromatösen Herden Kalkablagerungen, so sieht man diese im Röntgenbilde als isolierte herdweise Schatten bei sonst kaum sichtbarem Gefässe, so daß auch hierbei eine Verwechselung mit Mediaverkalkung in den meisten Fällen ausgeschlossen ist.

Die Fälle von Arteriosklerose der Extremitätengefäße betrafen (bis auf 2) Individuen, die das 50. Lebensjahr bereits überschritten hatten, so daß die Erkrankung vorwiegend als senile Endarteriitis chronica deformans bezeichnet werden konnte; dabei war das Verhältnis von Männern zu Frauen 18:12. Entsprechend der meist schweren Gefässerkrankung fanden sich bedeutend häufiger Folgezustände, als bei der reinen Mediaverkalkung. So konnten 3 mal große Aneurysmen an der Aorta konstatiert werden; 5 mal waren durch Erkrankung der Hirnarterien starke Veränderungen im Zentralnervensystem gesetzt; ebenso oft waren arteriosklerotische Schrumpfnieren, 3 mal Gangrän der Füße und 2 mal ausgedehnte Degenerationen des Myocards als Folgen der Gefäßveränderungen anzusehen. In 8 Fällen fand sich eine starke, in weiteren 9 eine mäßige Hypertrophie des linken Herzventrikels. 
Als Schlufsätze meiner Untersuchungen möchte ich folgende aufstellen:

1. Die Mediaverkalkung ist an den Arterien der Extremitäten sehr viel häufiger, als die Arteriosklerose.

2. Sind die Extremitätenarterien als "starre, geschlängelte, fragile Rohre" zu fühlen, so handelt es sich in der weitaus überwiegenden Mehrzahl der Fälle um eine Mediaverkalkung, nicht. um eine Arteriosklerose.

3. Weder aus dem Grade, noch aus der Ausdehnung der peripherischen Mediaverkalkung darf auf eine Arteriosklerose zentraler Gefäße ohne weiteres geschlossen werden; beide Erkrankungen finden sich häufig kombiniert, doch kommen Fälle hochgradigster Mediaverkalkung peripherischer Arterien vor ohne jegliche Arteriosklerose innerer Gefäße.

Zum Schlusse sei es mir gestattet, meinem hochverehrten Chef, Herrn Dr. Eugen Fraenkel, für die Anregung zu dieser Arbeit und für die Unterstützung bei derselben auch an dieser Stelle meinen besten Dank auszusprechen.

\section{Literatur.}

1. Marcband: Artikel Arterien in Eulenburg's Realencyclopädie, Bd. 2, 1994.

2. Orth: Lehrbuch der spez. patbol. Anatomie. Berlin 1887.

3. Strümpell: Lebrbuch der spez. Pathologie und Therapie. Leipzig 1897.

4. Ernst: Verhandlungen der Deutschen Pathol. Gesellsehaft zu Hamburg 1901.

5. Matusewicz: Isolierte Verkalkung der Elastica interna. Ziegl. Beitr., Bd. 31.

6. Dmitrijeff: Veränderung des elastischen Gewebes der Arterienwände bei Arteriosklerose. Ziegler's Beitr., Bd. 22, 1897.

7. Thoma: Über die Abbängigkeit der Bindegewebsneubildung der Arterienintima ron den mechanischen Bedingungen des Blutkreislaufs. Dies. Arch., Bd. 92, 95, 104, 105.

8. Mönckeberg: Über Knochenbildung in der Arterienwand. Dies. Arch. Bd. 167, 1902.

9. Cobn: Über Knochenbildung an den Arterien. Dies. Arch., Bd. 106, 1886.

10. Kaufmann: Lebrb. der spez. pathol. Anatomie, 2. Aufl., Berlin 1901. 
11. Küttner: Über Kalkmetastasen. Dies. Arch., Bd. 55 .

12. E. Fraenkel: Diskussion in der Sitzung der Biologischen Abteilung des ärztl. Vereins zu Hamburg vom 3. Dez. 1901.

13. Saenger: Über das intermittierende Hinken. Vortrag in derselben Sitzung.

\section{IX.}

Knochendeformationen bei einem Affen.

(Vergleichende Messungen an 6 Hapale-Skeletten, sowie kurze klinische Bemerkungen.)

Von

Dr. Carl Davidsohn,

Assistenten am Pathologischen Institut der Universität Berlin.

(Hierzu 4 Textfiguren.)

Der Vortrag von Hansemanns in der Berliner Medizinischen Gesellschaft am 12. Dezember $1900^{1}$ ) ließ sogleich das höchste Interesse erwachen, wie sich bei weiteren Untersuchungen die Knochen der Affen, die längere Zeit hier im nordischen Klima gelebt haben, verhalten würden; denn daß die Affektion wirklich Rachitis sei, identisch der unserer Berliner Kinder, wurde sowohl durch die ausgestellten Skelette als auch durch die Diskussion Virchows mindestens zweifelhaft.

Ich will das Skelett eines Affen zu beschreiben versuchen, der während des Lebens in ausgezeichneter Weise einige Symptome zeigte, die von Hansemann als rachitische anspricht; nach der Skelettierung ist jedoch ein so abweichendes Verhalten der krankhaft deformierten Knochen von der Rachitis zu erkennen, daß die Krankheit, wie Virchow meinte, eher als Osteomalacie aufzufassen, keinesfalls aber der Racbitis zuzuzählen wäre.

Aus der Lebensgeschichte des Tieres, das, obne zu wachsen, an seinem nur $11 \mathrm{~cm}$ langen Rumpfe einen $24 \mathrm{~cm}$ langen Schwanz hatte, will ich nur kurz das Wichtigste erwähnen.

1) v. Hansemann: Die Rachitis des Schädels, eine vergleicbend-anatomische Untersuchung. Berlin 1901. 